\title{
Searching for Extreme Mass-Ratio Binary Systems
}

\author{
Kevin Gullikson* \\ University of Texas at Austin \\ E-mail: kgulliks@astro.as.utexas.edu
}

Adam Kraus

University of Texas at Austin

Sarah Dodson-Robinson

University of Texas at Austin

\begin{abstract}
Most intermediate-mass stars are in binary or higher order multiple systems. However, the distributions of orbital parameters for systems with mass-ratios (the ratio of the primary to secondary masses) $q \lesssim 0.2$ are almost completely unknown due to the large flux ratio between the primary and secondary star. These extreme mass-ratio systems can provide information on the formation mechanism that the more "low hanging fruit" of equal mass binaries cannot. For instance, secondary stars formed as a result of gravitational instability in the disk of the young primary are expected to have $q \sim 0.1$. We present here initial results on a survey searching for low-mass companions to nearby A and B stars. We use high signal-to-noise ratio and high resolution spectroscopy to search directly for M or K star spectra within the noise of the primary star. This method is sensitive to low-mass stars that are on too wide of orbits to detect with traditional radial velocity monitoring, but too close of orbits to detect with imaging techniques. We find several new candidate companions, which produces a roughly flat mass-ratio distribution.
\end{abstract}

Frank N. Bash Symposium 2013: New Horizons in Astronomy

October 6-8, 2013

Austin, Texas

\footnotetext{
* Speaker.
} 
How do intermediate- and high-mass binary systems form? The primary mechanism is thought to be core fragmentation, where a collapsing molecular core develops two or more Jeans-mass overdensities that begin collapsing on their own. Many simulations of this process produce a mass-ratio distribution that is either flat or shows a preference for equal mass binaries (e.g. Bate et al. 2012). However, observations seem to hint that there is a slight propensity for very unequal mass systems amongst A- and late B-stars (Kouwenhoven et al. 2005). These low mass-ratio systems may have formed by gravitational instability in the protostellar disk. Simulations of disk fragmentation as a whole tend to produce lower mass-ratios than those of core fragmentation, and so we expect that an excess of low mass-ratio companions may indicate the importance of disk fragmentation.

We present here some initial results of a spectroscopic search of about 150 main sequence $A$ and B type stars. Rather than monitoring the radial velocity motion of the primary star, which has broad lines due to their large rotation speeds, we directly search the spectrum for the spectral lines of the secondary star. We have obtained high signal-to-noise and high-resolution echelle spectra of our sample stars using a variety of spectrographs, and have searched for companions by crosscorrelating the data against a grid of model spectra for GKM dwarf stars. This method is sensitive to lower-mass stellar companions than radial velocity monitoring can achieve at orbits $a \gtrsim 10 \mathrm{AU}$, which is inwards of the typical separation of similar adaptive optics surveys (e.g De Rosa et al. 2013, Kouwenhoven et al. 2005).

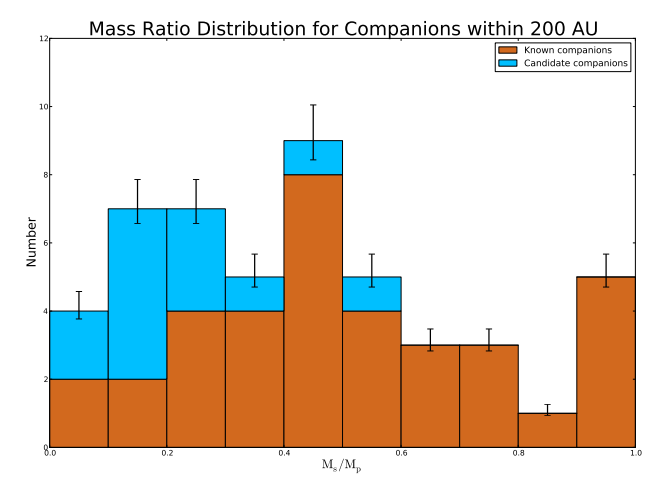

Figure 1: The mass-ratio distribution for our current sample of A and B stars, with known companions in orange and our new candidate companions in blue.

We have identified several new low-mass candidate companions in our survey. We show the mass-ratio distribution of our sample below, showing both our new companions and the previously known companions from the Ninth Catalog of Spectroscopic Binary Orbits (Pourbaix et al. 2004), the Washington Double Star Catalog (Mason et al 2013), and those given in Eggleton et al. (2008). This sample is about one half of our total sample, and so represents a work in progress. However, the current mass-ratio distribution appears quite flat, in agreement with the small separation mass-ratio distribution for field A-stars from De Rosa et al. (2013).

\section{References}

[1] Bate, M. R. Stellar, brown dwarf and multiple star properties from a radiation hydrodynamical simulation of star cluster formation 2012, MNRAS, 419, 3115 [ast ro-ph.SR/1110.1092]

[2] De Rosa, R. J. et al. The VAST Survey - III. The multiplicity of A-type stars within 75 pc 2013, MNRAS [astro-ph.SR/1311.7141]

[3] Eggleton, P. P. \& Tokovinin, A. A. A catalogue of multiplicity among bright stellar systems 2008, MNRAS, 389, 869 [astro-ph/0806.2878]

[4] Kouwenhoven, M. B. N. et al. The primordial binary population. I. A near-infrared adaptive optics search for close visual companions to A star members of Scorpius OB2 2005, A\&A, 430, 137 [astro-ph/ 0410106 ]

[5] Mason, B. D. et al. The Washington Visual Double Star Catalog (Mason+ 2001-2013) 2013, VizieR Online Data Catalog, 1, 2026

[6] Pourbaix, D. et al. The ninth catalogue of spectroscopic binary orbits 2004, A\&A, 424, 727 [astro-ph/0406573] 